\title{
ACERCA DEL DECIR PSICOANALÍTICO EN REVISTAS ARGENTINAS DE LOS AÑOS ‘50
}

\section{ABOUT THE PSYCHOANALYTIC SAYING IN ARGENTINE JOURNALS OF THE 50S}

\author{
Karina Savio ${ }^{l}$
}

Fecha de recepción: 9 de octubre de 2017 - Fecha de aceptación: 14 de noviembre de 2017

\begin{abstract}
Resumen
Enmarcado en los estudios del Análisis del Discurso, este artículo se propone investigar los modos en que el objeto discursivo psicoanálisis circula en diversas revistas argentinas durante la década del cincuenta. Las revistas suelen generar y difundir conocimientos, ideas, valores que repercuten en el imaginario social, por lo que su estudio permite un acercamiento al universo cultural de una sociedad en determinada época. En este trabajo, realizamos un recorrido por dos semanarios populares -El Hogar y Para ti- y dos publicaciones prestigiosas -Sur e Imago Mundi-y recopilamos, sistematizamos y analizamos aquellas zonas textuales en las que se hace referencia explícita tanto al psicoanálisis como a su fundador. Entre los resultados alcanzados, hallamos, en primer lugar, cierta correlación entre la aparición del término psicoanálisis y los períodos democráticos. En segundo lugar, evidenciamos un estrecho vínculo entre el psicoanálisis, la psicología aplicada y el peronismo. Como resultado del empuje que la psicología aplicada tiene durante el gobierno de Perón, advertimos que el psicoanálisis se filtra en diversos artículos. Observamos, por otra parte, la mención a la presencia de nociones psicoanalíticas en el lenguaje cotidiano y, finalmente, encontramos tres modalidades bajo las que el objeto psicoanálisis se construye: como un saber "maldito", como un saber sobre el inconsciente y como un saber "revolucionario”. Estas tres modalidades pueden ubicarse en tres momentos de la década. Mientras que la primera predomina en los primeros años, la segunda asoma a partir de 1953 aproximadamente y la tercera se fortalece durante el último tiempo.

Palabras clave: Análisis del discurso, Objeto discursivo, Peronismo, Historia del psicoanálisis,
\end{abstract} Discurso.

\begin{abstract}
Based on the studies of Discourse Analysis, this article aims to investigate the ways in which the discursive object psychoanalysis circulates in several Argentine magazines during the fifties. Magazines often generate and disseminate knowledge, ideas, values that impact on the social imaginary, so that their study allows an approach to the cultural universe of a society at a determined time. In this work, we examine two popular weeklies - El Hogar y Para ti - and two prestigious publications -Sur and Imago Mundi - to compile, systematize and analyze those textual areas in which explicit reference is made both to psychoanalysis and to its founder. Among the results achieved, we find, first, a certain correlation between the emergence of the term psychoanalysis and democratic periods. Second, we observe a close link between psychoanalysis, applied psychology and the peronism. As a result of the push that applied psychology has during the administration of Perón, we note that psychoanalysis is filtered in different articles. We also note the mention of the presence of psychoanalytic notions in everyday language and, finally, we find three modalities under which the object psychoanalysis is constructed: as a "cursed" knowledge, as a knowledge about the unconscious and as a "revolutionary" knowledge. These three
\end{abstract}

1 Investigadora en CONICET, Docente en la Universidad Nacional Arturo Jauretche y Universidad de Buenos Aires, Argentina, karinasavio@fibertel.com.ar 
modalities can be located in three moments of the decade. While the first one predominates in the first years, the second one appears after 1953 approximately and the third one strengthens during the last time.

Keywords: Discourse Analysis, Discursive object, Peronism, Psychoanalysis history, Discourse.

\section{Introducción}

Desentrañar los motivos por los cuales el psicoanálisis penetró de una manera tan profunda en los distintos ámbitos de la sociedad argentina no es tarea sencilla. El alcance y la valoración que este saber ha obtenido en este país no pueden ser atribuidos, en verdad, a un único factor, a una única causa, sino a un complejo entramado de acontecimientos y circunstancias que impulsaron y edificaron esta irreversible instalación. Las investigaciones que se han ocupado de rastrear y, a su vez, construir una historia posible del psicoanálisis argentino son, hoy en día, numerosas y coinciden en ubicar el comienzo de este protagonismo en los años sesenta, década en la que la figura del psicoanalista logra visibilidad pública (Balán, 1991; Dagfal, 2009; Plotkin, 2003; Vezzetti, 1996, 1999, entre otras). En ese entonces, un público ampliado accede a la lectura de Freud y, como señala Terán (2004, p.75), el lenguaje psicoanalítico penetra "en revistas populares, shows televisivos, obras de teatro, ficción y ensayo".

Ahora bien, la relación entre los medios de comunicación y este saber, previa a este boom, ha sido un tema que ha quedado relativamente al margen de estos trabajos, a pesar de su importancia en la divulgación de este discurso (Plotkin, 2006; Savio, 2016, en prensa; Vezzetti, 2012). En efecto, en el período anterior a esos años, la mayoría de los estudios suele detenerse en los vaivenes de la fundación de la Asociación Psicoanalítica Argentina (APA) y en el lugar que ocupa el psicoanálisis en diferentes espacios institucionales relativos a la salud y a la universidad, sin considerar la cuestión de su difusión.

En estas páginas nos proponemos abordar, entonces, la presencia del psicoanálisis en diversas revistas argentinas durante la década del cincuenta con el fin último de observar e interpretar el modo o los modos que adopta esta manifestación. Partimos de entender a las revistas como un "emprendimiento destinado a captar/influenciar a un determinado sector de la sociedad -adulto, infantil, femenino- que se siente atraído por las temáticas más diversas: política, espectáculos, arte, humor, deportes, historia, literatura, entre otras" (Panella y Korn, 2010, p. 9). Por tal motivo, generan y difunden conocimientos, ideas, valores que repercuten en el imaginario social y en el universo cultural de una sociedad. Para este artículo hemos revisado las publicaciones de El Hogar, Para ti, Sur e Imago Mundi de esos años y hemos recopilado, sistematizado y analizado aquellas zonas textuales en las que se menciona explícitamente tanto el psicoanálisis como a su fundador.

Antes de comenzar a desarrollar esta problemática, es importante destacar que durante la década del cincuenta y, fundamentalmente, durante sus primeros cinco años la psicología recibe un gran impulso por parte del Estado. Klappenbach (2006) describe este período como el período de la psicotecnia y de la orientación profesional, dado que en la Argentina se elabora un conjunto de teorías, técnicas e intervenciones prácticas relativas a estos campos del conocimiento. A lo largo del primer peronismo el rol clave que juega la psicología aplicada, funcional a los proyectos del gobierno, se refleja, por un lado, en la creación de diversas instituciones y carreras que se ocupan de estos estudios y, por el otro, en la realización del Primer Congreso Argentino de Psicología, llevado a cabo en Tucumán en $1954^{2}$. En este escenario, Dagfal (2009) afirma que el psicoanálisis “oficial”, es decir, el de la Asociación Psicoanalítica Argentina, se mantiene a distancia de las transformaciones socioculturales que sacuden al país. No obstante, es necesario precisar el alcance de esta afirmación. En este sentido, el saber psicoanalítico se hace visible en el primer congreso, que es respaldado por el Estado: de hecho, un grupo de psicoanalistas,

2 Para una ampliación de este tema véase Dagfal $(2012,2014)$ 
entre ellos, Ángel Garma, participa del evento y expone diversos trabajos (Gentile, 1997) ${ }^{3}$. Y, por otra parte, como veremos más adelante, el empuje que cobra la psicología en estos primeros años contribuye a que el psicoanálisis se cuele -de diferentes maneras- en el discurso social.

En este artículo, luego de plantear nuestra perspectiva teórico-metodológica, desplegaremos brevemente, en un primer momento, los rasgos que caracterizan las revistas seleccionadas y los géneros discursivos por los que transita el discurso psicoanalítico. Intentaremos construir aquí el itinerario que recorre tomando en cuenta los años de publicación, los temas abordados y los autores -en los casos pertinentes-. En una segunda instancia, exploraremos el vínculo entre el lenguaje y el psicoanálisis. Nos encontraremos, en este punto, con que algunos términos psicoanalíticos ya forman parte del lenguaje cotidiano a principios de la década analizada. Finalmente, revelaremos tres modos bajo los que se manifiesta el psicoanálisis en los materiales trabajados: como un saber "maldito", como el saber del inconsciente y como un saber "revolucionario".

\section{Perspectiva teórico-metodológica}

Este trabajo forma parte de una investigación mayor en la que se busca reflexionar sobre el lugar que ocupa el psicoanálisis en diversos medios de comunicación en la década del cincuenta. Enmarcada dentro del Análisis del Discurso (AD), en particular, su línea francesa, el estudio pretende entender qué se concibe como discurso psicoanalítico y bajo qué modalidades circula en materiales seleccionados de esos años. Consideramos que las revistas ofrecen un escenario interesante de análisis ya que son portavoces de las ideas, valores y creencias de su tiempo e intervienen en la configuración del espacio simbólico de una sociedad.

En esta investigación planteamos dos líneas esenciales para el análisis. En primer lugar, el discurso, en nuestro caso el discurso psicoanalítico, no puede pensarse en términos absolutos, como un discurso anterior. Siguiendo a Courtine (1981), sostenemos que es un objeto a construir: no prexiste al investigador, sino que es este quien le da cierta consistencia imaginaria. En segundo lugar, esta construcción no es un conjunto cerrado. En efecto, su naturaleza cambiante y su heterogénea constitución impiden que sea aprehendido en su integridad. Por ello, manifestamos que no existe un discurso psicoanalítico, sino que conviven y convergen en él diferentes entramados discursivos.

Para este artículo, nos interesa estudiar el psicoanálisis como un objeto de discurso y del discurso, esto es, un saber sobre el que se predica. El término objeto fue introducido por Foucault (2005, p.73) en La arqueología del saber, en donde define la formación discursiva como el sistema de reglas que forman los objetos, las modalidades enunciativas, los conceptos y las estrategias presentes en los enunciados. Esta definición conlleva un implícito: los objetos no están dados de antemano, sino que se construyen a partir de ciertas "reglas de formación". En este texto, el autor señala, además, que estos objetos no pueden formarse en cualquier momento de la historia, sino que son necesarias ciertas condiciones para que puedan surgir y para que se pueda "decir algo" de ellos: "no se puede hablar en cualquier época de cualquier cosa; no es fácil decir algo nuevo" (2005, p.73). Otro punto indispensable para entender estos objetos es que están inmersos en un haz complejo de relaciones establecidas entre instituciones, procesos económicos y sociales, formas de comportamiento, sistemas de normas, técnicas, modos de caracterización, que son las que les permiten aparecer.

El AD adopta, a su vez, esta noción de objeto, al que entiende en términos de interdiscurso; en efecto, plantea que los objetos discursivos se forman en el espacio interdiscursivo. Si bien en Las verdades evidentes (2016) Pechêux no nombra este término, afirma que el interdiscurso conecta y articula elementos discursivos constituidos por él como un preconstruido, o sea, como elementos anteriores a lo construido en y por el enunciado. Estos elementos pueden leerse, en verdad, como objetos. Así lo

3 Para una ampliación acerca de la relación entre psicoanálisis y el peronismo véase Argañaraz (2007). 
hace Courtine (1981), quien aclara que es el interdiscurso el lugar en el que se constituyen, para un sujeto hablante productor de una secuencia discursiva dominada por una formación discursiva dada, los objetos de los que el sujeto enunciador se apropia para hacerlos objetos de su discurso, como así de las articulaciones entre estos objetos ${ }^{4}$.

En las siguientes páginas, entonces, nos proponemos indagar en torno a los modos en que se forma el objeto discursivo psicoanálisis. Por esta razón, en los artículos publicados en las revistas $E l$ Hogar (EH), Para ti (PT), Sur (S) e Imago Mundi (IM) durante la década del cincuenta ubicamos los enunciados en los que aparece el término psicoanálisis como en los que se hace referencia a Sigmund Freud. Buscamos observar, de esta forma, cuáles son los sentidos que se construyen discursivamente sobre este objeto $^{5}$.

\section{De revistas y géneros}

Antes de comenzar a desplegar el análisis realizado, es necesario recordar aquí, brevemente, algunas de las características que singularizan las revistas elegidas. Debemos tener en cuenta que estas publicaciones difieren, entre otras particularidades, en cuanto a sus perfiles, sus objetivos, la cantidad de ejemplares publicados y el público al que están destinadas. En términos generales, podemos destacar que Para ti y El Hogar son dos semanarios populares, orientados, en principio, a un sector amplio de la sociedad, mientras que Sur e Imago Mundi son revistas culturales, dirigidas a un público "ilustrado", más restringido.

Para $t i$-primera revista femenina del país- se edita por primera vez el 16 de mayo de 1922 por la editorial Atlántida y publica cuentos, sociales, actualidad y consejos para la mujer sobre moda y belleza, entre otros temas ${ }^{6}$. El primer número de El Hogar, publicado por la editorial de Alberto Haynes, sale, por su parte, en 1904 y, aunque podría ser pensada como una revista para la mujer ya que incluye secciones femeninas, apunta, en verdad, a toda la familia, por lo que incorpora una gran cantidad de artículos de interés general ${ }^{7}$. Sus lectores pertenecen a los estratos medios-altos y altos de la sociedad. Diferentes son los casos de Imago Mundi y Sur. La primera de ellas, dedicada a la historia de la cultura y dirigida por José Luis Romero, comienza a publicarse trimestralmente en septiembre de 1953 y se edita por tres años. En su producción participa un grupo de intelectuales que mantiene diferencias con el peronismo ${ }^{8}$. Sur, fundada por Victoria Ocampo, es una revista literaria emblemática del país, referente de la cultura argentina, que ve la luz en 1931. Esta revista aspira a la promoción y difusión de obras y de ideas, pero también a la consolidación de un grupo intelectual. Su último número aparece en 1992, trece años después de la muerte de la escritora?.

Del relevamiento que hemos realizado en torno a estas cuatro revistas, quisiéramos establecer dos señalamientos que son significativos para nuestro estudio y que están en relación con los años en los que surge el término psicoanálisis y los géneros en los que se lo incluye.

Con respecto a los años en los que se hace referencia al psicoanálisis, advertimos que su frecuencia de aparición es mayor durante el peronismo. En efecto, entre 1950 y septiembre de 1955 hallamos catorce textos en los que se lo nombra. Entre fines de 1955 y mayo de 1958, es decir, entre el golpe cívico-militar y la presidencia de Frondizi, ubicamos cinco artículos. Finalmente, en 1959 se publican

\footnotetext{
4 Para una ampliación sobre objetos del discurso, se puede leer a Arnoux $(2006,2008)$.

5 Detallamos los textos hallados en estas revistas al final de este artículo.

6 Sobre los inicios de Para ti véase Bontempo (2011), Eujanian (1999).

$7 \quad$ Sobre la revista El Hogar véase Eujanian (1999).

8 Sobre la revista Imago Mundi véase Acha (1999, 2007).

9 Sobre la revista Sur existe una gran cantidad de bibliografía publicada. Véase, por ejemplo, King (1989).
} 
tres. La correlación entre los gobiernos democráticos y la alusión a este nuevo saber, y su notable presencia en el peronismo pueden reflejarse, en particular, en la revista El Hogar: a pesar de que empieza a obtener gradualmente una mayor visibilidad, el término psicoanálisis es citado por última vez a fines del ' 55 y recién vuelve a mencionarse en 1959. Estos primeros datos nos permiten ir anticipando el cruce que puede establecerse entre el alcance que -como ya hemos recordado- logra el campo de la psicología en el peronismo y el impulso que el saber psicoanalítico recibe en los medios. Podría pensarse, en este sentido, que el auge de la psicología de aquellos años es una variable que participa, junto a otras, en la promoción y en la divulgación de la teoría psicoanalítica en el espacio social.

Respecto a los lugares en los que esta noción es incluida, de la lectura del corpus se desprende que la circulación del término psicoanálisis no se restringe a un único género, sino que, por el contrario, se extiende a lo largo de diversas formas discursivas.

En la revista Para ti, por ejemplo, se publica un cuento del escritor español Francisco Valle de Juan en 1950 bajo ese título. En este sentido, el psicoanálisis se constituye aquí como marco ficcional en el que se inscribe la historia.

En El Hogar el término aparece en una crónica social, en una nota de opinión, en una reseña, en artículos periodísticos, en una entrada de diccionario especializado y en entrevistas. Con respecto a estas últimas, entre 1953 y 1954 en la Tribuna de la revista -cuya centralidad se refleja en que aparece en las páginas tres y cuatro del semanario- se publica una serie de entrevistas a diferentes personalidades que versan sobre la psicología: Juan José Berruezo, Carlos Pourteau Agote, Oscar Oñativia, Ricardo Moreno, Pedro Badanelli, José Dick y Jorge Marcelo David. Los temas tratan sobre la psicología en general, sus áreas de aplicación -medicina psicosomática, psicología laboral, psicología forense, entre otras- y también sobre el Primer Congreso Argentino de Psicología que se realiza en Tucumán en 1954. Estos textos, en los que se describe a la psicología como la "ciencia de moda" (EH, 2305, 1954, p.3) visibilizan el alcance que adquiere este domino del saber durante el peronismo. Por otra parte, en cuanto a los autores de los demás artículos encontramos a Linda Lindsay, Franco Pierini y Ulises Barrera, quienes ejercieron como periodistas, y a dos escritores: Thorp McClusky y Luisa Sofovich ${ }^{10}$. Será recién en 1959 cuando aparecerá un psicoanalista en escena: frente a la famosa separación de los actores Leigh y Olivier se convoca la opinión de diversas personalidades argentinas, entre ellas, al psicoanalista Arnaldo Rasckovsky. Las temáticas que se abordan en estos textos son múltiples: abarcan desde la vida sentimental de Freud hasta la relación entre el psicoanálisis y la moda.

En Imago Mundi, a pesar de ser una revista de historia, se publica en 1956 un ensayo titulado "La crisis contemporánea según Jung" por Marcos Victoria, un médico que será el primer director de la carrera de psicología de la Universidad de Buenos Aires, en el que se hace referencia al psicoanálisis.

Finalmente, en Sur la noción circula en ensayos, reseñas bibliográficas, una conferencia y la traducción de un artículo de Jung. Entre los que escriben, además de este último psicoanalista, están: el periodista Alberto Zalamea, el filósofo Julián Marías, el escritor Enrique Anderson Imbert, el médico Raúl Ballbé, y los psicoanalistas León Ostrov y Eduardo Krapf. El único psicoanalista argentino que figura en estos años y que escribe especialmente para esta revista es, entonces, Ostrov. Sin embargo, su texto nace a raíz de un comentario que publica Zalamea a propósito del libro Freud, el mago sexual de Emil Ludwig en el que se realiza una fuerte crítica al psicoanálisis. La nota del psicoanalista polemiza con el contenido de la reseña y destaca las virtudes de este saber. La psiquiatría, el arte y la esquizofrenia son algunos de los temas que se tratan en estos artículos, sobre los que el psicoanálisis puede aportar

10 Algunos de los artículos se publican sin firma. 


\section{El lenguaje y el psicoanálisis}

A pesar de que -como ya hemos afirmado en la introducción- los estudios coinciden en indicar que el psicoanálisis se populariza en la Argentina en la década del sesenta, varios artículos que se publican a comienzos del cincuenta muestran la temprana incidencia de este saber, en particular, de algunas de las nociones que forman parte de su armazón teórica, en el lenguaje cotidiano. En efecto, estos textos revelan que algunos términos provenientes del psicoanálisis son adoptados tempranamente en el decir de los argentinos.

Esta relación, la del lenguaje y el psicoanálisis, no es percibida en los primeros años como una relación conveniente o fructífera. Por el contrario, algunos artículos denuncian no solo el "(ab)uso" de términos psicoanalíticos en el habla corriente, sino también, la generalización y, por ende, la deformación del empleo de ciertas categorías con las que los sujetos se identifican.

1. Hasta en el lenguaje corriente ha trascendido la influencia del freudismo (...) Está de moda tener complejos (...). Todos los fracasos por falta de simpatía, de talento, de constancia, de bondad o de fortuna, se atribuyen hoy al demonio invisible de un complejo ${ }^{11}$. (PT, 1441, 1950, p.27)

Este fragmento corrobora la transferencia del vocabulario psicoanalítico a la vida diaria, y el auge y la aceptación que adquiere, en este caso, la utilización de la noción de "complejo". No obstante, este alcance -que está acompañado de cierta jactancia- permite, según esta cita, la justificación de todos los fracasos, por lo que se lo caracteriza como un "demonio invisible".

En 1952 otro artículo refiere también a este singular problema: la propagación de este conocimiento contribuye a su banalización. Veamos un recorte:

2. Esos vocablos [subconsciente, complejos, libido], que en otros tiempos suponían conceptos muy solemnes reservados a la cátedra científica y a los textos (...) hoy se han vulgarizado de tal manera, mejor dicho, se han abaratado tanto, que ya no son un índice de cultura ni mucho menos. (EH, 2217, 1952, p.12)

El pasaje del psicoanálisis del acotado e ilustre recinto científico a la sociedad "salvaje" no es sin consecuencias. Su divulgación conlleva una pérdida, su utilización ya no es marca de prestigio. El saber psicoanalítico es ahora un saber popular, un saber de las masas, por el que es posible excusar los comportamientos:

3. Ahora, en cambio, cualquier chico de tercer grado, en el mismo trance [frente a un aplazo], en cuanto le preguntamos (...) Nos contesta lo más orondo:

-No...jme vino el complejo! $!^{12}$ (EH, 2217, 1952, p.12)

Al igual que en el primer ejemplo, el complejo, que adquiere en la respuesta del estudiante cierta autonomía en su modo de aparición, funciona aquí como escudo protector que borra culpas y responsabilidades.

Ahora bien, es en 1953 el año en el que se publica una entrevista en la que el vínculo entre el lenguaje y el psicoanálisis se presenta, por primera vez, despojado de su cara negativa. En una entrevista sobre psicología aplicada se plantea el uso de términos psicoanalíticos en el habla; sin embargo, esta utilización no es percibida como un descrédito.

11 A menos que se aclare los destacados son nuestros.

12 El destacado pertenece al original. 
4. Difícilmente encontraremos quien no hable de sus "complejos" o de los ajenos. Este término, que pertenecía hasta no hace mucho al psicoanalista, está ahora en todos los labios. Se quiere significar con esta palabra "diagnóstico" cuanto ocurre de anormal en la esfera subjetiva. $(E H, 2275,1953$, p.3)

En esta cita no se problematiza la incorporación de estas nociones en el entramado social, ya que las palabras adquieren en el habla un nuevo sentido.

El lenguaje psicoanalítico no solamente es abordado en estos textos en su relación con la oralidad, sino también con la escritura y, en especial, con la escritura de Freud. Encontramos también en torno a este asunto dos posturas bien delimitadas. Mientras que en 1952 en la revista Sur se hace alusión a la "mediocridad" de la escritura freudiana, en 1954 en la revista El Hogar se pondera el estilo de este autor:

5. la paradoja de un gran descubrimiento científico expresado en forma totalmente anticientífica por su autor (...) Los escritos de Freud son, efectivamente, mediocres (S, 207-208, 1952, p.126)

6. Como escritores (...) sabíamos que después de él no solo se escribe de otra manera (...) es bien cierto que siempre, antes y después de Freud, el escritor y el artista geniales (EH, 2324, 1954, p.28, p.112)

En el ejemplo cinco se contraponen el descubrimiento freudiano y su forma de transmisión. En este sentido, los hallazgos no condicen con la manera en la que se los comunica. En contraposición a esta postura, en el ejemplo seis Freud es caracterizado como un "gran escritor" y se le atribuye, a partir de su trabajo, un cambio en el modo de escribir.

\section{El objeto discursivo psicoanálisis}

De la lectura y del análisis de los materiales, nos encontramos con que el objeto discursivo psicoanálisis se construye en torno a tres sentidos: como un saber "maldito", como un saber sobre el inconsciente y como un saber "revolucionario". Estas tres modalidades no deben ser interpretadas como modalidades cerradas y absolutas; son, en verdad, modos discursivos que en los diversos artículos se entremezclan y se entrecruzan. No obstante, hallamos que, durante los primeros años, el primer sentido, el psicoanálisis "maldito", cobra una mayor visibilidad y predomina en el entramado discursivo, para, luego, ir cediendo paulatinamente espacio a la segunda y a la tercera vertiente. En los próximos subapartados desplegaremos más acabadamente estas variantes.

\section{a. El psicoanálisis "maldito"}

En varios de los artículos que se publican entre 1950 y 1952 el psicoanálisis es presentado desde una doble lectura. Por un lado, se lo concibe como un saber novedoso al que se le reconoce "veracidad" y legitimidad; por el otro, se le adjudica un carácter "nocivo" para la sociedad. Y será esta última propiedad aquella que se privilegie. En otras palabras, en estos textos, se producen dos movimientos simultáneos: se admite el aporte de la teoría freudiana del inconsciente al saber, al estudio de la psiquis, pero se pone el foco fundamentalmente en el daño que esta teoría generó y/o genera en el espacio social. El psicoanálisis se presenta así como un "prejuicio" $(E H, 2217,1952$, p.111) que acarrea "terribles consecuencias" ( $E H, 2217,1952$, p.12). Esta vertiente subsiste, aunque en menor intensidad, en algunos de los artículos posteriores. Veamos cómo se muestra esta dualidad:

7. Sus estudios, que realmente tuvieron una base seria, fueron bien pronto desvirtuados por las exageraciones a que llegaron sus discípulos, alentados por el propio Freud, que perdió los estribos en alas de su fantasía de sexómano. (EH, 2217,1952, p.12) 
En el ejemplo se visualiza la oposición entre estas dos posiciones: la "base seria" de los estudios, por una parte, y las "exageraciones", por la otra. Es interesante leer cómo esto se atribuye a la condición de "sexómano" de Freud.

En cuanto al carácter pernicioso del psicoanálisis, en estos artículos se observa, en primer lugar, cierta isotopía estilística, característica del discurso religioso. Diversos términos -como "sagrado" (EH, 2217, 1952, p.111) o "alma" (S, 207-208, 1952, p.126) entre varios otros- trazan un campo semántico cercano a este discurso. Como efecto de sentido, se produce, en estos casos, una homologación entre el psicoanálisis y las creencias religiosas.

8. Y aquel hombre, "el más guapo de los dos", curaba los espíritus enfermos, reparaba los cerebros averiados, daba nueva fe a los que habían perdido la suya, infundía paciencia a los que la hubieran gastado esperando verse libres de sus obsesiones. (PT, 1441, 1950, p.8)

9. En la nueva religión encontraron un dogma (a ninguno le fue permitido discutir y modificar las ideas del maestro), un ritual (el diván donde yace el paciente, con la silla para el sicoanalista y las tres o cuatro sesiones semanales), un jefe (el respeto por la figura de Freud para los sicoanalistas ortodoxos es muy cercano a la idolatría). (EH, 2577, 1959, p.33)

En el ejemplo ocho se utiliza un vocabulario característico del discurso de la religión. En este enunciado los quehaceres que se vinculan con la labor del psicoanalista, protagonista del cuento, son análogos a los de un profeta; recuerdan, incluso, a la figura de Jesús y su predicación. Por su parte, en el nueve esta homologación queda explicitada: aquí se hace referencia al psicoanálisis como una “nueva religión”. A partir de esta descripción, se introducen varias críticas, ya que implica un dogma, un ritual y un jefe.

Al igual que en la discursividad religiosa, en la que abundan figuras retóricas relacionadas con el par antitético luz-oscuridad, en estos artículos se incorporan metáforas y comparaciones vinculadas con la oscuridad. En este sentido, la religión psicoanalítica postula un inconsciente que podríamos describir como "tenebroso" y "sombrío".

10. La subconsciencia viene a ser el sótano de nuestro temperamento (...) a ese lugar maldito (...) Pues va a caer, directamente, como una piedra, en el pozo oscuro de lo subconsciente (PT, 1441, 1950, p.27)

En este fragmento encontramos dos metáforas que reenvían a esta antinomia: "sótano" y "oscuro". El inconsciente -que es nombrado en estos textos como "subconsciente"- es incluso concebido como un "lugar maldito".

En segundo lugar, en estos artículos se vislumbra una tensión entre el discurso psicoanalítico y el discurso moral. Recordemos que los desarrollos psicoanalíticos que interrogan la sexualidad y, fundamentalmente, la postulación de una sexualidad infantil son dos cuestiones que producen un significativo rechazo por parte de la comunidad académica en la época freudiana. Retomando esta línea, en algunos de los textos que conforman nuestro corpus, se polemiza con las teorías de Freud en tanto cuestionan los preceptos morales y, de esta manera, las normas sociales que dictaminan y distinguen entre el "bien" y el "mal". En otras palabras, el psicoanálisis es contemplado como un saber que se opone a los valores, las costumbres, las creencias que guían los comportamientos y que diferencian lo correcto de lo incorrecto. Si el psicoanálisis es una religión que estudia lo oscuro, lo maldito, entonces, su saber agujerea la moral instituida.

11. Porque el autor del psicoanálisis, en otro exceso no menos absurdo, pretendió situar todos los males de la psiquis humana dentro de un solo campo: el del sexo. (EH, 2217, 1952, p.12)

12. los principios de la lógica freudiana, amén de antinaturales y falsos, resultan profundamente inmorales y constituyen un sistema realmente nocivo y hasta peligroso por la sociedad. (EH, 2217, 1952, p.111) 
En el fragmento once al sexo, entendido como causa de todos los "males", se le califica como un "exceso". El segundo ejemplo se refiere a la inmoralidad de los desarrollos freudianos y su peligrosidad de manera explícita.

Ahora bien, este "exceso" en la teoría psicoanalítica, asociado a la sexualidad infantil, es adjudicado, en dos de las notas, a la historia personal de Freud y a sus propios problemas psicológicos:

13. así también el creador del psicoanálisis, investigador de manías ajenas, se delató asimismo como un maniático flagrante, obsesionado por la sexomanía. (EH, 2217, 1952, p.12)

14. Fromm pone en evidencia que ni siquiera el mismo Freud había captado el sentido exacto de su intenso amor por la madre, y expone el parecer de que el descubrimiento del fundamental complejo de Edipo sea debido más a su situación personal de relaciones filiales que a la intuición racional de una nueva teoría científica. $(E H, 2577,1959$, p. 30)

En el recorte trece Freud es diagnosticado como "maniático" y como "sexómano". En el catorce se le atribuye la invención del complejo de Edipo a su cercana relación con su madre.

Finalmente, en estos artículos se hace mención en varias oportunidades a otra de las polémicas que nace en el surgimiento del psicoanálisis y que también continúa vigente en la actualidad: el cuestionamiento de su valor científico. Esta crítica, emparentada con la idea de que este saber, en verdad, es análogo a una creencia religiosa, figura en algunos de estos artículos en los que se duda respecto de la rigurosidad de la teoría analítica. De esta forma, lo que subyace a estos comentarios es que el psicoanálisis no es una ciencia.

15. estos casos inclinan a pensar más bien en que nos hallamos frente al charlatanerismo, lisa y llanamente. (EH, 2217, 1952, p.111)

16. El talón de Aquiles del psicoanálisis es, pues, la carencia de pruebas. (S, 207-208, 1952, p.127)

En estos dos ejemplos adquiere cuerpo la posición que esgrime la falta de cientificidad de este saber. El primero se refiere al "charlatanerismo" y el segundo, a la ausencia de "pruebas".

\section{b. El psicoanálisis como saber del inconsciente}

A partir de 1953, el psicoanálisis se presenta, en algunos de los textos, como un saber entre saberes: es el saber que se dedica al estudio del inconsciente. Esta visión circula con fuerza en las entrevistas que tratan acerca de los diversos aspectos sobre los que trabaja la psicología y sobre sus distintas áreas de incumbencia, pero también en algunos de los otros artículos, en los que se incorpora la palabra de algún psicoanalista como cita de autoridad. Podría pensarse que esta variante es una variante que se construye desde un registro más moderado: ni se acusa al psicoanálisis de dañar la sociedad, ni se lo exalta como un saber sagrado y único.

El psicoanálisis se configura así como una teoría que puede ser estudiada, analizada, e, incluso, criticada desde una posición más neutral. No obstante, es importante destacar que aquello que se valora está en relación, fundamentalmente, con el inconsciente y con el sueño. La libido, la sexualidad infantil, la pulsión de muerte, el complejo de Edipo, entre otras, son nociones que ponen en cuestión, como señalábamos, los preceptos morales de aquella época, por lo que quedan por fuera de estos discursos, que se ciñen, entonces, a aquello "decible", aquello que puede ser aceptado por la sociedad.

Como ya hemos recordado, durante los años en los que gobierna el peronismo la psicología ocupa un rol clave en el ámbito público y estatal. En ese entonces, en donde se advierte un auge de este campo de estudio, la psicología es entendida como una disciplina "moderna" que atraviesa numerosas dimensiones del entramado social. Es interesante observar, por ende, que, a pesar de que en aquellos tiempos se privilegia la psicología aplicada, el psicoanálisis se cuela en el decir de los entrevistados, quienes reconocen su mérito y contribución al conocimiento. 
17. Es importante destacar que los progresos de la psicoterapia y de la neurología y, sobre todo, las técnicas de exploración del inconsciente aportadas por el psicoanálisis abrieron a la psicología un campo maravilloso e insospechado. $(E H, 2305,1954$, p.4)

18. La psicología jurídica, cuya base y orientación tiene que ser eminentemente biológicas, apoyándose especialmente en el conductismo, en la personalogía, en la psicología de la forma, en el psicoanálisis, en el sprangerianismo, en la medicina legal, en la psiquiatría en incluso en la metapsíquica. (EH, 2324, 1954, p.4)

En el ejemplo diecisiete se alude a cómo las técnicas de "exploración" del inconsciente, junto a otros estudios, contribuyeron al desarrollo de la psicología. En el recorte siguiente, en el que se aborda la problemática de la psicología forense, se observa que el psicoanálisis es ubicado como una teoría más en una serie en la que se incluyen múltiples paradigmas de los estudios psicológicos.

Este saber, como afirmamos al comienzo, es un saber con el que se puede discutir y polemizar. En algunas entrevistas, si bien se resalta el valor de los hallazgos freudianos, se cuestionan o se problematizan algunos de sus planteos teóricos. En este sentido, el psicoanálisis no se erige como una verdad única y absoluta, sino como otra herramienta para el análisis. Este es el caso, por ejemplo, de una entrevista sobre medicina psicosomática en la que se realza el lugar del psicoanálisis para su desarrollo, pero, luego, se limita su alcance:

19. El psicoanálisis es, sin duda, uno de los pilares de la medicina psicosomática. La medicina moderna debe a Freud el estudio de la vida anímica, con originales técnicas exploratorias. El notable hombre de ciencia vienés ha contribuído [sic] como ningún otro a esclarecer la influencia fundamental de los factores psicógenos en la génesis de enfermedades otrora desconocidas. $(E H, 2323,1954$, p.4)

En este fragmento se establece que el saber psicoanalítico es "uno" de los pilares de la medicina psicosomática y, con subjetivemas como "notable" y "originales", se elogian tanto su estudio como a su fundador. Sin embargo, más adelante se sostiene:

20. Si bien es cierto que reconocemos el ingente aporte del psicoanálisis (...), no podemos comulgar, (...), en considerar únicamente los factores psicógenos en la interpretación de las enfermedades y someter a éstas a los rígidos moldes de la psicoterapia analítica. $(E H, 2323,1954$, p.4)

A partir de una concesiva, vemos que en este fragmento se le concede al psicoanálisis un aporte fundamental para el trabajo clínico, pero se le adjudica, simultáneamente, una estructura "rígida" con la que se distancia.

Además de construirse como un campo de saber, el discurso psicoanalítico emerge, en algunos de los artículos en los que el psicoanálisis no es el tópico central, como cita de autoridad desde donde argumentar o reflexionar sobre algún tema puntual. De esta manera, las voces de algunos psicoanalistas se incorporan en estos textos dialogando con otras voces u otras posiciones que contribuyen a pensar determinado asunto:

21. En efecto, el eminente médico vienés Freud (1856-1940), conocido universalmente como el padre del psicoanálisis, afirmaba que la vida psíquica del individuo yace en lo inconsciente, y confesaba que la existencia del genio humano era para él "un enigma psicológico". (EH, 2255, 1953, p.16)

El ejemplo pertenece a un artículo en el que se interroga si los genios poseen o no poderes sobrenaturales. El autor nutre el planteo de esta nota a partir de la palabra de escritores, compositores, científicos, filósofos y, además, con esta referencia a Freud. En este fragmento se enfatiza la importancia y el prestigio "universal" del padre del psicoanálisis y, a partir de una cita mixta, se señala su lectura sobre el inconsciente y sobre la genialidad de los seres humanos. 


\section{c. El psicoanálisis revolucionario}

Aparte de los dos modos en que el psicoanálisis se concibe en estos artículos -como psicoanálisis "maldito" y como saber del inconsciente- se evidencia una tercera modalidad que atraviesa toda la década, pero que se irá consolidando para 1959: el psicoanálisis como saber "revolucionario". En este sentido, en algunos textos se advierte una tercera mirada en la que se enaltecen, desde un lenguaje hiperbólico, tanto las propiedades del psicoanálisis como las cualidades y virtudes de su fundador. A pesar de que se podría sospechar, en un primer momento, que esta dimensión se restringe únicamente a las notas firmadas por psicoanalistas, consta también de artículos escritos por autores de diversas procedencias.

La analogía entre el descubrimiento freudiano y la revolución aparece explícitamente en múltiples lugares. Esta visión conlleva a leer este hallazgo como un cambio radical y profundo respecto a la forma en que se entiende la vida psíquica, pero también implica inscribirlo en términos bélicos:

22. Por ejemplo, en la Ilíada se encuentra una interpretación muy parecida, guardando la distancia de los tiempos, de la que Freud nos ha dado del inconsciente revolucionando la psicología y psiquiatría modernas. $(E H, 2304,1954$, p.4)

23. le dió[sic] la posibilidad de modificar nuestra imagen del hombre de manera realmente revolucionaria. $(S, 217-218$, 1956, p.48)

En estos dos ejemplos se observa con claridad la caracterización del psicoanálisis como revolucionario: revolucionó la psicología y la psiquiatría, y la imagen misma del ser humano.

Esta revolución es, por otra parte, llevada adelante por el padre del psicoanálisis, Sigmund Freud, a quien se engrandece y se elogia en numerosas notas y también en algunas de las entrevistas:

24. los que ayudarían a Freud en su genial construcción. (EH, 2324, 1954, p.28)

25. en gran parte es obra de un solo hombre. (S, 211-212, 1952, p.146)

En el primer ejemplo se realza la imagen de Freud, dado que, mediante el uso de un subjetivema evaluativo axiológico, se refiere a su "genial" elaboración. Es interesante ver cómo la cita veinticinco contribuye al carácter épico y, podríamos añadir, heroico del trabajo de freudiano. En efecto, en este enunciado se aclara que el psicoanálisis es obra de "un solo hombre".

Esta exaltación de la figura del vienés se traslada, a su vez, a su pensamiento y a su construcción teórica, que son calificados, polemizando con la posición que afirma que el psicoanálisis no es una ciencia, como científicos. En la respuesta de León Ostrov a la reseña escrita por Zalamea encontramos, por ejemplo, los siguientes puntos:

26. Es la de Freud la actitud ejemplar del hombre de ciencia. (S, 211-212, 1952, p.143)

27. Las hipótesis que Freud formula responden a las rigurosas exigencias que el espíritu científico supone: verificabilidad e inteligibilidad. (S, 211-212, 1952, p.143)

En contraposición a lo que Zalamea destaca en torno a la carencia de pruebas de la teoría psicoanalítica, Ostrov nombra a Freud como un "hombre de ciencia" y emplea términos que reenvían a las cualidades de exactitud y formalidad propias del método científico. De esta forma, lo que se quiere destacar es que el psicoanálisis es, efectivamente, una ciencia.

El campo semántico religioso, al que nos hemos referido previamente y que es utilizado para cuestionar este saber, se presenta también en estos artículos, pero, en este caso, se invierte su sentido. Mientras que en la primera vertiente, aquella que nombramos como psicoanálisis "maldito" se establece una analogía entre este discurso y la religión como un modo de objetar sus bases teóricas, esta visión se vale de ella para elogiar sus propiedades. 
28. Es hombre casado, con una hija ya casada. Sabe, pues, lo que es un hogar. Y por lo tanto, además de médico de almas en privado, en un secreto recinto que tiene la sagrada garantía del confesionario (...) sabe también cuánto puede significar su destrucción, el desmoronamiento de una familia, que significa todo divorcio. $(E H, 2585,1959$, p.72)

En el fragmento, a partir del empleo de un léxico propio del discurso religioso, se nombra al psicoanalista como "médico de almas" y al consultorio como "la sagrada garantía del confesionario".

Por último, quisiéramos indicar que en el año 1959, frente al conocido caso Leigh-Olivier se incorpora la palabra de Arnaldo Rasckovsky, psicoanalista de la Asociación Psicoanalítica Argentina, y se lo presenta de la siguiente manera:

29. El doctor Arnaldo Rasckovsky, sicoanalista cuya fama rebasa las fronteras del país, se inició en la práctica de su profesión como clínico de niños. (EH, 2585, 1959, p.72)

En el ejemplo se observa que ya en este año, por un lado, el psicoanálisis es un discurso prestigioso y legítimo en la sociedad argentina y, por el otro, se especifica que la fama de Rasckovsky trasciende el país.

\section{Conclusiones}

En este artículo nos ha interesado ahondar en un tema que ha sido poco explorado hasta el momento y que está en relación con el modo o los modos en que el discurso psicoanalítico circula en los medios de comunicación durante la década del cincuenta, antes de que se produzca el conocido boom psicoanalítico en la década del sesenta. Para ello, estudiamos diversas revistas, con distintos perfiles y, por ende, con diferentes lectores, que se publican en esos años: dos semanarios populares -Para ti y El Hogar- y dos revistas prestigiosas, destinadas a un público más restringido -Imago Mundi y Sur-. Luego del recorrido propuesto y de la lectura realizada, podemos precisar algunas de las conclusiones a las que hemos arribado.

En primer lugar, este trabajo evidencia que en los años cincuenta, incluso ya desde sus inicios, el psicoanálisis es un discurso presente en estas revistas. Podemos suponer que tanto la teoría psicoanalítica como su fundador, Sigmund Freud, forman parte, aunque todavía no de una manera consolidada, del acervo cultural de ese entonces. Asimismo, en algunos de estos artículos se deja entrever que en el lenguaje cotidiano ya figuran algunas de las nociones psicoanalíticas, tales como complejo o inconsciente.

En segundo lugar, el análisis ha permitido correlacionar la aparición de textos que refieren a este campo o a Freud con los años de democracia. En este sentido, se observa que durante los períodos democráticos el psicoanálisis cobra una mayor fuerza, fundamentalmente, en la revista El Hogar. Esta distinción no es tan nítida en la revista Sur, aunque es interesante señalar que en los comienzos de la década se percibe un mayor número de artículos relativos a esta temática.

Otra derivación significativa del trabajo está vinculada al estrecho lazo que puede entablarse entre el discurso psicoanalítico, la psicología y el peronismo. Como resultado del empuje que la psicología aplicada tiene durante el gobierno de Perón, advertimos que el psicoanálisis se filtra, quizás fortuitamente, en la revista El Hogar, lo que permite cimentar gradualmente su posición en el entramado social. Podría pensarse, entonces, que la emergencia del psicoanálisis es un efecto, entre otras variables, del impulso que recibe la psicología.

Por último, encontramos que el objeto discursivo psicoanálisis está construido en torno a tres sentidos: como saber "maldito", como saber del inconsciente y como saber "revolucionario". Estas tres modalidades pueden ubicarse en tres momentos de la década. Mientras que la primera predomina en los primeros años, la segunda asoma a partir de 1953 aproximadamente y la tercera se fortalece durante el último tiempo (1958-1960). Es interesante destacar aquí que tanto la clínica psicoanalítica como 
aquellas nociones que entran en tensión con la moral de la época quedan por fuera del entramado discursivo. En efecto, el psicoanálisis aparece en estos años como una teoría del sueño y del inconsciente.

\section{Referencias}

Acha, Omar (1999). Imago Mundi (1953-1956) en una coyuntura historiográfico política. Prismas, Revista de historia intelectual, $\mathrm{N}^{\circ} 3,117-142$.

Acha, Omar (2007). Revistas de las afueras del peronismo: Contorno e Imago Mundi entre la renovación historiográfica y el proyecto generacional. En Guillermo Korn (Comp.), El peronismo clásico (1945-1955). Descamisados, gorilas y contreras (pp.239-251), Buenos Aires: Paradiso Ediciones.

Argañaraz, Juan de la Cruz (2007). El freudismo reformista. 1926-1976. En La literatura y la medicina, la política y la psicología. Córdoba: Brujas.

Arnoux, Elvira (2006). Análisis del discurso. Modos de abordar materiales de archivo. Buenos Aires: Santiago Arcos.

Arnoux, Elvira (2008). Los discursos sobre la nación y el lenguaje en la formación del Estado (Chile, 1842-1862). Estudio glotopolítico. Buenos Aires: Santiago Arcos.

Balán, Jorge (1991). Cuéntame tu vida. Una biografía colectiva del psicoanálisis argentino. Buenos Aires: Planeta Espejo de la Argentina.

Bontempo, Paula (2011). Para Ti: una revista moderna para una mujer moderna, 1922-1935. Estudios Sociales, 41, 127-156.

Courtine, Jean-Jacques (1981). Quelques problèmes théoriques et méthodologiques en analyse du discours, à propos du discours communiste adressé aux chrétiens. Langage, 15 (62), 9-128.

Dagfal, Alejandro (2009). Entre París y Buenos Aires. La invención del psicólogo (1942-1966). Buenos Aires: Paidós.

Dagfal, Alejandro (2012). Historias de la psicología en la Argentina (1890-1966). Entre ciencia natural y disciplina del sentido. Ciencia Hoy, vol. 21, 126, pp. 21-25.

Dagfal, Alejandro. (2014). Breve historia de la psicología en la ciudad de La Plata (1906-1966). Universitas Psychologica, 13(5), 1759-1775.

Eujanian, Alejandro (1999). Historia de revistas argentinas. 1900-1950. La conquista del público. Buenos Aires: Asociación Argentina de Editores de Revistas.

Foucault, Michel (2005). La arqueología del saber. Buenos Aires: Siglo veintiuno.

Gentile, Antonio (1997). El Primer Congreso Argentino de Psicología. 1954. Cuadernos Argentinos de Historia de la Psicología, 3 (1/2), pp. 159-173.

King, John (1989). Sur. Estudio de la revista argentina y de su papel en el desarrollo de una cultura (1931-1970). México: F.C.E.

Klappenbach, Hugo (2006). Periodización de la psicología en Argentina. Revista de Historia de la Psicología, vol. 27, núm.1, 109-164.

Panella, Claudio y Korn, Guillermo. (2010) Introducción. En Panella, Claudio y Korn Guillermo (comp.) Ideas y debates para la nueva Argentina. Revistas culturales y políticas del peronismo (19461955) (pp.9-13), Vol. I, La Plata: Universidad Nacional de La Plata.

Pecheux, Michel (2016). Las verdades evidentes: lingüística, semántica, filosofía. Ciudad Autónoma de Buenos Aires: Ediciones del Centro Cultural de la Cooperación Floreal Gorini.

Plotkin, Mariano (2003). Freud en las Pampas. Buenos Aires: Sudamericana.

Plotkin, Mariano (2006). El psicoanálisis antes del boom. En Biagini, H. y A. Roig (dir.) El pensamiento alternativo en la Argentina del siglo XX: obrerismo y justicia social (1930-1960) (pp. 519-541). Buenos Aires: Biblos.

Savio, Karina (2016). Acerca de los inicios de la divulgación del psicoanálisis en Argentina: un estudio de Escuela para Padres. Literatura y Lingüística, 33, 95-128.

Savio, Karina (en prensa). Saber, práctica, sujeto: el consultorio psicoanalítico en la revista Idilio. Letras. 
Terán, Oscar (2004). Ideas e intelectuales en la Argentina, 1880-1980. En Terán, Oscar (coord.) Ideas en el siglo. Intelectuales y cultura en el siglo XX latinoamericano (pp.13-95). Buenos Aires: Siglo XXI Editores Argentina.

Vezzetti, Hugo (1996). Aventuras de Freud en el país de los argentinos. De José Ingenieros a Enrique Pichón-Rivière. Buenos Aires: Paidós.

Vezzetti, Hugo (1999). Las promesas del psicoanálisis en la cultura de masas. En Historia de la vida privada en la Argentina. Tomo III (pp.173-197). Buenos Aires: Taurus.

Vezzetti, Hugo (2012). El psicoanálisis y los sueños en Idilio. En Priamo, Luis. Sueños. Fotomontajes de Grete Stern. Serie completa. Edición de la obra impresa en la revista Idilio (1948-1951) (pp.149159). Buenos Aires: Fundación CEPPA.

\section{Artículos analizados}

Para ti

1950

- $\quad$ No1441, 17 de enero, "Psicoanálisis", por Francisco Valle de Juan, pp. 8,9, 27, 28, 32.

$$
\text { El Hogar }
$$

1951

- $\quad$ № 2197, 21 de diciembre, "Juventud, arquitectura y psicoanálisis”, por Linda Lindsay, El Hogar, pp. $71,142$.

1952

- $\quad \mathrm{N}^{\circ} 2217,9$ mayo, “¿Fue Freud un gran embaucador?”, pp.12, 111.

1953

- $\quad$ N²255, 30 de enero, “¿Qué es la medicina psicosomática?”, pp.3-4, 75.

- $\quad \mathrm{N}^{\circ} 2255,30$ de enero, “Poseen los genios poderes sobrenaturales?”, por Thorp McClusky, pp.16-17.

- $\quad N^{\circ} 2275,19$ de junio, “Qué procura la psicología aplicada a la actividad profesional?”, pp.3-4, 87. 1954

- $\quad \mathrm{N}^{\circ} 2304,8$ de enero, “LLa psicología es, como se dice, una ciencia moderna?", pp.3-4, 140.

- $\quad \mathrm{N}^{\circ} 2305,15$ de enero, “Algo más sobre psicología: ¿cuál es su aspecto principal?”, pp. 3-4, 72.

- $\quad \mathrm{N}^{\circ} 2323,21$ de mayo, "El concepto de 'cuerpo y alma' en la medicina moderna”, pp.3-4, 73.

- $\quad \mathrm{N}^{\circ} 2324,6$ de agosto, “¿Qué es la psicología jurídica o forense?”, pp. 3-4.

- $\quad N^{\circ} 2352,10$ de diciembre, “QQué es la logoterapia o el análisis existencial?”, pp.3-4, 140.

- $\quad N^{\circ} 2351,3$ de diciembre, "Sigmund Freud, enamorado", por Luisa Sofovich, pp.28, 112.

1955

- $\quad \mathrm{N}^{\circ} 2398,4$ de noviembre, "Pequeño diccionario filosófico para materialistas", p.106. 1959

- $\quad$ N²577, 24 de abril, "Freud sobre el diván”, Franco Pierini, pp.29-33.

- $\quad \mathrm{N}^{\circ} 2585,19$ junio, "El teléfono llamó diez veces", pp.71-74.

- $\quad \mathrm{N}^{\circ} 2585,19$ junio, "Los conflictos en el matrimonio", por Ulises Barrera, pp.26-27.

Imago Mundi

1956

- ํ11-12, marzo-junio, “La crisis contemporánea según Jung”, por Marcos Victoria, pp.208-211. Sur

1952

- $\quad$ N²07-208, enero-febrero, "Reseña de Freud, el mago sexual de Emil Ludwig”, por Alberto Zalamea, pp.125-128.

- $\quad$ N²11-212, mayo-junio, “A propósito de una nota sobre ‘Freud, el mago sexual”, por León Ostrov, pp.143-147. 
- $\quad$ N²17-218, noviembre-diciembre, "La psiquiatría vista desde la psicología”, por Julián Marías, pp.11-26.

- $\quad N^{\circ} 217-218$, noviembre-diciembre, “Leonardo y Freud”, por Enrique Anderson Imbert, pp.64-66. 1956

- $\quad$ No217-218, septiembre-octubre, "El significado del psicoanálisis para nuestra imagen del hombre”, por Enrique Anderson Imbert, pp.46-51.

- N²17-218, septiembre-octubre, "El Marcos Victoria: Freud, Jung, Adler", por Raúl Ballbé, pp.76-80. 1957

- $\quad$ N²46, mayo-junio, “La mente del hombre”, por Carl Jung, pp.4-8. 
